\title{
Almost Unbiased Estimator for Estimating Population Mean Using Known Value of Some Population Parameter(s)
}

\author{
Rajesh Singh \\ Department of Statistics, Banaras Hindu University (U.P.), India \\ rsinghstat@yahoo.com \\ Mukesh Kumar \\ Department of Statistics, Banaras Hindu University (U.P.), India \\ mukesh.stat@gmail.com \\ Florentin Smarandache \\ Department of Mathematics, University of New Mexico, Gallup, USA \\ smarand@unm.edu
}

\begin{abstract}
In this paper we have proposed an almost unbiased estimator using known value of some population parameter(s). Various existing estimators are shown particular members of the proposed estimator. Under simple random sampling without replacement (SRSWOR) scheme the expressions for bias and mean square error (MSE) are derived. The study is extended to the two phase sampling. Empirical study is carried out to demonstrate the superiority of the proposed estimator.
\end{abstract}

Key words: Auxiliary information, bias, mean square error, unbiased estimator, two phase sampling.

\section{Introduction}

Consider a finite population $U=U_{1}, U_{2}, \ldots U_{N}$ of $N$ units. Let $y$ and $x$ stand for the variable under study and auxiliary variable respectively. Let $\left(y_{i}, x_{i}\right), i=1,2, \ldots, n$ denote the values of the units included in a sample $s_{n}$ of size $n$ drawn by simple random sampling without replacement (SRSWOR). The auxiliary information has been used in improving the precision of the estimate of a parameter (See Cochran (1977), Sukhatme et. al. (1984) and the references cited there in). Out of many methods, ratio and product methods of estimation are good illustrations in this context.

In order to have a survey estimate of the population mean $\bar{Y}$ of the study character $\mathrm{y}$, assuming the knowledge of the population mean $\overline{\mathrm{X}}$ of the auxiliary character $\mathrm{x}$, the well-known ratio estimator is

$$
\mathrm{t}_{\mathrm{r}}=\overline{\mathrm{y}} \frac{\overline{\mathrm{x}}}{\overline{\mathrm{x}}}
$$

Bahl and Tuteja (1991) suggested an exponential ratio type estimator as -

$$
\mathrm{t}_{\mathrm{re}}=\overline{\mathrm{y}} \exp \left[\frac{\overline{\mathrm{X}}-\overline{\mathrm{x}}}{\overline{\mathrm{X}}+\overline{\mathrm{x}}}\right]
$$


Several authors have used prior value of certain population parameter(s) to find more precise estimates. Sisodiya and Dwivedi (1981), Sen (1978) and Upadhyaya and Singh (1984) used the known coefficient of variation (CV) of the auxiliary character for estimating population mean of a study character in ratio method of estimation. The use of prior value of coefficient of kurtosis in estimating the population variance of study character y was first made by Singh et. al. (1973). Later used by Singh and Kakaran (1993) in the estimation of population mean of study character. Singh and Tailor (2003) proposed a modified ratio estimator by using the known value of correlation coefficient. Kadilar and Cingi (2006), Khosnevisan et. al. (2007), Singh et. al. (2007) Singh and Kumar (2009) and Singh et. al. (2009) have suggested modified ratio estimators by using different pairs of known value of population parameter(s).

In this paper under SRSWOR, we have proposed almost unbiased estimator for estimating $\overline{\mathrm{Y}}$.

\section{Almost unbiased ratio type estimator}

Suppose

$\mathbf{t}_{0}=\bar{y}, \quad t_{r s}=\bar{y}\left(\frac{a \bar{x}+b}{\bar{x}+b}\right), \quad t_{r s e}=\bar{y} \exp \left\{\frac{(a \bar{x}+b)-(a \bar{x}+b}{(a \bar{x}+b)+(a \bar{x}+b}\right\}$

Such that $t_{0}, t_{r s}, t_{r s e} \in w_{r}$ where $w_{r}$ denotes the set of all possible ratio type estimators for estimating the population mean $\overline{\mathrm{Y}}$. By definition the set $\mathrm{w}_{\mathrm{r}}$ is $\mathrm{a}$ linear variety, if

$t_{w r}=\omega_{0} \bar{y}+\omega_{1} t_{r s}+\omega_{2} t_{r s e} \in w$

for $\quad \sum_{i=0}^{2} \omega_{i}=1, \quad \omega_{i} \in \mathrm{R}$

where $\omega_{i}(i=0,1,2)$ denotes the statistical constants and $R$ denotes the set of real numbers.

To obtain the bias and MSE of $t_{w}$, we write

$\overline{\mathrm{y}}=\overline{\mathrm{Y}}\left(1+\mathrm{e}_{0}\right), \quad \overline{\mathrm{X}}=\overline{\mathrm{X}}\left(1+\mathrm{e}_{1}\right)$,

such that

$$
\begin{aligned}
& E\left(e_{0}\right)=E\left(e_{1}\right)=0 . \\
& E\left(e_{0}^{2}\right)=f_{1} C_{y}^{2}, \quad E\left(e_{1}^{2}\right)=f_{1} C_{x}^{2}, \quad E\left(e_{0} e_{1}\right)=f_{1} \rho C_{y} C_{x} . \\
& \text { where } f_{1}=\left(\frac{1}{n}-\frac{1}{N}\right), \quad S_{y}^{2}=\frac{1}{(N-1)} \sum_{i=1}^{N}\left(y_{i}-\bar{Y}\right)^{2}, \quad S_{x}^{2}=\frac{1}{(N-1)} \sum_{i=1}^{N}\left(x_{i}-\bar{X}\right)^{2}, \\
& C_{y}=\frac{S_{y}}{Y}, C_{x}=\frac{S_{x}}{X}, \quad K=\rho\left(\frac{C_{y}}{C_{x}}\right), \rho=\frac{S_{y x}}{\left(S_{y} S_{x}\right)}, \\
& S_{y x}=\frac{1}{(N-1)} \sum_{i=1}^{N}\left(y_{i}-\bar{Y}\right)\left(x_{i}-\bar{X}\right) .
\end{aligned}
$$


Expressing $t_{w}$ in terms of e's, we have

$\mathbf{t}_{\mathrm{w}}=\overline{\mathrm{Y}}\left(1+\mathrm{e}_{0}\right)\left[\omega_{0}+\omega_{1}\left(1+\theta \mathrm{e}_{1}\right)^{-1}+\omega_{2} \exp \left\{-\frac{\theta \mathrm{e}_{1}}{2}\left(1+\theta \mathrm{e}_{1}\right)^{-1}\right\}\right]$

where $\theta=\frac{\mathrm{a} \overline{\mathrm{X}}}{\mathrm{a} \overline{\mathrm{X}}+\mathrm{b}}$.

Expanding the right hand side of (2.3) and retaining terms up to second order of e's, we have

$\mathbf{t}_{\mathrm{w}} \cong \overline{\mathrm{Y}}\left[1+\mathrm{e}_{0}-\omega \theta \mathrm{e}_{1}+\theta^{2}\left(\omega_{1}+\frac{3}{8}\right) \mathrm{e}_{1}^{2}-\theta \omega \mathrm{e}_{0} \mathrm{e}_{1}\right]$

where $\omega=\left(\omega_{1}+\frac{\omega_{2}}{2}\right)$.

Taking expectations of both side of (2.4) and then subtracting $\bar{Y}$ from both side, we get the bias of the estimator $t_{w}$, up to the first order of approximation as

$\mathbf{B}\left(\mathrm{t}_{\mathrm{w}}\right)=\mathrm{f}_{1} \overline{\mathrm{Y}}\left[\theta^{2} \mathrm{C}_{\mathrm{x}}^{2}\left(\omega_{1}+\frac{3 \omega_{2}}{8}\right)-\theta \omega \rho \mathrm{C}_{\mathrm{y}} \mathrm{C}_{\mathrm{x}}\right]$

$B\left(t_{r s}\right)=f_{1} \bar{Y}\left[\theta^{2} C_{x}^{2}-\theta \rho C_{y} C_{x}\right]$

$B\left(t_{\text {rse }}\right)=f_{1} \bar{Y}\left[\frac{3 \theta^{2} C_{\mathrm{x}}^{2}}{8}-\frac{\theta \rho C_{y} C_{x}}{2}\right]$

From (2.4), we have

$\left(\mathrm{t}_{\mathrm{w}}-\overline{\mathrm{Y}}\right) \cong \overline{\mathrm{Y}}\left[\mathrm{e}_{0}-\theta \omega \mathrm{e}_{1}\right]$

Squaring both sides of (2.9) and then taking expectations, we get MSE of the estimator $t_{w}$, up to the first order of approximation, as

$\operatorname{MSE}\left(\mathrm{t}_{\mathrm{w}}\right)=\mathrm{f}_{1} \overline{\mathrm{Y}}\left[\mathrm{C}_{\mathrm{y}}^{2}+\theta^{2} \omega^{2} \mathrm{C}_{\mathrm{x}}^{2}-2 \theta \omega \rho \mathrm{C}_{\mathrm{y}} \mathrm{C}_{\mathrm{x}}\right]$

This is minimum when

$\omega=\mathrm{k}\left(=\rho \frac{\mathrm{c}_{\mathrm{y}}}{\mathrm{c}_{\mathrm{x}}}\right)$.

Putting this value of $\omega(=k)$ in (2.10), we get the minimum MSE of $\mathbf{t}_{\mathrm{w}}$ as

$\min . \operatorname{MSE}\left(t_{w w}\right)=f_{1} \bar{Y}^{2} C_{y}^{2}\left(1-\rho^{2}\right)$

which is same as that of traditional linear regression estimator from (2.5) and (2.11), we have

$\omega_{1}+\frac{\omega_{2}}{2}=\mathrm{k}$

From (2.2) and (2.13), we have only two equations in three unknowns. It is not possible to find the unique values for $\omega_{i^{\prime} s}, i=0,1,2$. In order to get unique values for $\omega_{i^{\prime} s}$, we shall impose the linear restriction

$\omega_{0} \mathrm{~B}(\overline{\mathrm{y}})+\omega_{1} \mathrm{~B}\left(\mathrm{t}_{\mathrm{rg}}\right)+\omega_{2} \mathrm{~B}\left(\mathrm{t}_{\mathrm{rse}}\right)=0$

Equations (2.2), (2.11) and (2.14) can be written as in the matrix form as

$\left[\begin{array}{ccc}1 & 1 & 1 \\ 0 & 1 & 1 / 2 \\ 0 & \mathrm{~B}\left(\mathbf{t}_{\mathrm{rs}}\right) & \mathrm{B}\left(\mathrm{t}_{\mathrm{rse}}\right)\end{array}\right]\left[\begin{array}{l}\omega_{0} \\ \omega_{1} \\ \omega_{2}\end{array}\right]=\left[\begin{array}{l}1 \\ k \\ 0\end{array}\right]$ 
Using (2.15), we get unique values of $\omega_{i^{\prime} 8}(i=0,1,2)$ as

$\left.\begin{array}{l}\omega_{0}=\frac{\Delta_{0}}{\Delta_{\mathrm{r}}} \\ \omega_{1}=\frac{\Delta_{1}}{\Delta_{\mathrm{r}}} \\ \omega_{2}=\frac{\Delta_{2}}{\Delta_{\mathrm{r}}}\end{array}\right\}$

where

$$
\left.\begin{array}{c}
\Delta_{\mathrm{r}}=\mathrm{B}\left(\mathrm{t}_{\mathrm{rse}}\right)-\frac{1}{2} \mathrm{~B}\left(\mathrm{t}_{\mathrm{rs}}\right) \\
\Delta_{\mathrm{r} 0}=\mathrm{B}\left(\mathrm{t}_{\mathrm{rse}}\right)\{1-\mathrm{k}\}+\frac{1}{2} \mathrm{~B}\left(\mathrm{t}_{\mathrm{rs}}\right)\left\{\mathrm{k}-\frac{1}{2}\right\} \\
\Delta_{\mathrm{r}}=\mathrm{k} \cdot \mathrm{B}\left(\mathrm{t}_{\mathrm{rse}}\right) \\
\Delta_{\mathrm{r} 2}=-\mathrm{kB}\left(\mathrm{t}_{\mathrm{rs}}\right)
\end{array}\right\}
$$

Use of these $\omega_{i^{\prime} \mathrm{g}}(\mathrm{i}=0,1,2)$ remove the bias up to terms of order $\left(\mathrm{n}^{-1}\right)$ at $(2.1)$.

\section{Product -type estimators}

Suppose $t_{0}=\bar{y}, \quad t_{p s}=\bar{y}\left(\frac{a \bar{x}+b}{a \bar{X}+b}\right), \quad t_{p s e}=\bar{y} \exp \left\{\frac{(a \bar{x}+b)-(a \bar{X}+b)}{(a \bar{x}+b)+(a \bar{X}+b)}\right\}$

such that $t_{0}, t_{p s}, t_{p s e} \in Q$, where $Q$ denotes the set of all possible product -type estimators for estimating the population mean $\bar{Y}$. By definition, the set $Q$ is linear variety if

$\mathrm{t}_{\mathrm{q}}=\mathrm{q}_{0} \overline{\mathrm{y}}+\mathrm{q}_{1} \mathrm{t}_{\mathrm{ps}}+\mathrm{q}_{2} \mathrm{t}_{\mathrm{pse}} \in \mathrm{Q}$

for $\sum_{i=0}^{2} q_{i}=1, \quad q_{i} \in R$

where $\mathrm{q}_{\mathrm{i}}(\mathrm{i}=0,1,2)$ denotes the statistical constants.

Expressing $\mathrm{t}_{\mathrm{q}}$ in terms of e's, we have

$\mathbf{t}_{\mathrm{q}}=\overline{\mathrm{y}}\left(1+\mathrm{e}_{0}\right)\left[\omega_{0}+\omega_{1}\left(1+\theta \mathrm{e}_{\mathbf{1}}\right)+\omega_{2} \exp \left\{\frac{\theta \mathrm{e}_{1}}{2}\left(1+\theta \mathrm{e}_{1}\right)^{-1}\right\}\right]$

where $\theta=\frac{a \bar{x}}{a \bar{X}+b}$.

Expanding the right hand side of (3.3) and retaining terms up to second power of e's, we have

$\mathbf{t}_{\mathrm{q}} \cong \overline{\mathrm{Y}}\left[1+\mathrm{e}_{0}+\theta \mathrm{qe}_{1}-\frac{\mathrm{q}_{2}}{8} \mathrm{e}_{1}^{2}+\mathrm{q} \theta \mathrm{e}_{\mathrm{o}} \mathrm{e}_{1}\right]$

where $\mathrm{q}=\mathrm{q}_{1}+\frac{\mathrm{q}_{2}}{2}$

Taking expectations of both sides of (3.4) and then subtracting $\bar{Y}$ from both sides, we get the bias of the estimator $\mathrm{t}_{\mathrm{q}}$, up to the first order of approximation as

$B\left(t_{\mathrm{q}}\right)=f_{1} \bar{Y}\left[-\frac{q_{z}}{8} \theta^{2} C_{x}^{2}+q \theta \rho C_{y} C_{x}\right]$ 
Bias expression for the estimators $t_{p s}$ and $t_{p s e}$ is given by

$$
\begin{aligned}
& \mathbf{B}\left(\mathrm{t}_{\mathrm{ps}}\right)=\mathrm{f}_{1} \overline{\mathrm{Y}}\left[\theta \rho \mathrm{C}_{\mathrm{y}} \mathrm{C}_{\mathrm{x}}\right] \\
& \mathbf{B}\left(\mathrm{t}_{\mathrm{pse}}\right)=\mathrm{f}_{1} \overline{\mathrm{Y}}\left[-\frac{1}{8} \theta^{2} \mathrm{C}_{\mathrm{x}}^{2}+\frac{\theta \rho \mathrm{c}_{\mathrm{y}} \mathrm{c}_{\mathrm{x}}}{2}\right]
\end{aligned}
$$

From (3.4), we have

$$
\left(\mathrm{t}_{\mathrm{q}}-\overline{\mathrm{Y}}\right) \cong \overline{\mathrm{Y}}\left[\mathrm{e}_{0}+\theta \mathrm{q} \mathrm{e}_{1}\right]
$$

Squaring both the sides of (3.9) and then taking expectations, we get MSE of the estimator $t_{\mathrm{q}}$, up to the first order of approximation, as

$\operatorname{MSE}\left(\mathrm{t}_{\mathrm{q}}\right)=\mathrm{f}_{1} \overline{\mathrm{Y}}^{2}\left[\mathrm{C}_{\mathrm{y}}^{2}+\theta^{2} \mathrm{q}^{2} \mathrm{C}_{\mathrm{x}}^{2}+2 \theta \mathrm{q} \rho \mathrm{C}_{\mathrm{y}} \mathrm{C}_{\mathrm{x}}\right]$

which is minimum for

$$
\mathrm{q}=-\mathrm{k}=-\rho \frac{\mathrm{c}_{\mathrm{y}}}{\mathrm{c}_{\mathrm{x}}}
$$

Putting this value of $q(=-k)$ in (3.10), we get the minimum MSE of $t_{q}$ as

$\min \operatorname{MSE}\left(t_{\mathrm{q}}\right)=\mathrm{f}_{1} \overline{\mathrm{Y}}^{2} \mathrm{C}_{\mathrm{y}}^{2}\left(1-\rho^{2}\right)$

which is same as that of traditional linear regression estimator.

From (3.5) and (3.11), we have

$\mathrm{q}_{1}+\frac{\mathrm{q}_{2}}{2}=-\mathrm{k}$

From (3.2) and (3.13), we have only two equations in three unknowns. It is not possible to find the unique values for $q_{i}{ }^{\prime} s, i=0,1,2$. In order to get unique values of $\mathrm{q}_{i}$ 's, we shall impose the linear restriction

$$
\mathrm{q}_{0} \mathrm{~B}(\overline{\mathrm{y}})+\mathrm{q}_{1} \mathrm{~B}\left(\mathrm{t}_{\mathrm{ps}}\right)+\mathrm{q}_{2} \mathrm{~B}\left(\mathrm{t}_{\mathrm{pse}}\right)=0
$$

Equations (3.2),(3.13) and (3.14) can be written in the matrix form as

$$
\left[\begin{array}{ccc}
1 & 1 & 1 \\
0 & 1 & 1 / 2 \\
0 & \mathrm{~B}\left(\mathrm{t}_{\mathrm{ps}}\right) & \mathrm{B}\left(\mathrm{t}_{\mathrm{pse}}\right)
\end{array}\right]\left[\begin{array}{l}
\mathrm{q}_{0} \\
\mathrm{q}_{1} \\
\mathrm{q}_{2}
\end{array}\right]=\left[\begin{array}{c}
1 \\
-\mathrm{k} \\
0
\end{array}\right]
$$

Solving (3.15), we get the unique values of $q_{i}{ }^{\prime} s(i=0,1,2)$ as-

$$
\left.\begin{array}{l}
\mathrm{q}_{0}=\frac{\Delta_{\mathrm{p} 0}}{\Delta_{\mathrm{p}}} \\
\mathrm{q}_{1}=\frac{\Delta_{\mathrm{p} 1}}{\Delta_{\mathrm{p}}} \\
\mathrm{q}_{2}=\frac{\Delta_{\mathrm{p} 2}}{\Delta_{\mathrm{p}}}
\end{array}\right\}
$$

where 


$$
\left.\begin{array}{c}
\Delta_{\mathrm{p}}=\mathrm{B}\left(\mathrm{t}_{\mathrm{pse}}\right)-\frac{1}{2} \mathrm{~B}\left(\mathrm{t}_{\mathrm{ps}}\right) \\
\Delta_{\mathrm{p} 0}=\mathrm{B}\left(\mathrm{t}_{\mathrm{pse}}\right)\{1+\mathrm{k}\}+\mathrm{B}\left(\mathrm{t}_{\mathrm{ps}}\right)\left\{-\mathrm{k}-\frac{1}{2}\right\} \\
\Delta_{\mathrm{p} 1}=-\mathrm{k} \cdot \mathrm{B}\left(\mathrm{t}_{\mathrm{pse}}\right) \\
\Delta_{\mathrm{p} 2}=\mathrm{kB}\left(\mathrm{t}_{\mathrm{ps}}\right)
\end{array}\right\}
$$

Use of these qi's $(i=0,1,2)$ remove the bias up to terms of order $o\left(n^{-1}\right)$ at $(3.1)$.

In Appendix A we have listed some of the important known estimators of the population mean, which can be obtained by suitable choice of constants $\omega_{\mathrm{i}}, \mathrm{i}=0,1,2, \mathrm{q}_{\mathrm{i}}, \mathrm{i}=0,1,2$ and $\mathrm{a}$ and $\mathrm{b}$.

\section{Proposed estimators in two phase sampling}

When $\overline{\mathrm{X}}$ is unknown, it is sometimes estimated from a preliminary large sample of size $n$ ' on which only the characteristic $x$ is measured (for details see Singh et. al. (2007)). Then a second phase sample of size $n\left(n<n^{\prime}\right)$ is drawn on which both $y$ and $x$ characteristics are measured. Let $\bar{x}=\frac{1}{n^{\prime}} \sum_{i=1}^{n^{\prime}} x_{i}$ denote the sample mean of $\mathrm{x}$ based on first phase sample of size n', $\bar{y}=\frac{1}{n} \sum_{i=1}^{n} y_{i}$ and $\bar{x}=\frac{1}{n} \sum_{i=1}^{n} x_{i}$, be the sample means of $y$ and $x$ respectively based on second phase of size $n$.

In two phase sampling the estimator $t_{w r}$ will take the following form

$t_{w d}=\omega_{0 d} \bar{y}+\omega_{1 d} t_{r s d}+\omega_{2 d} t_{r s e d} \in w_{d}$

for $\sum_{\mathrm{i}=0}^{2} \omega_{\mathrm{id}}=1, \quad \omega_{\mathrm{id}} \in \mathrm{R}$

where $t_{r s}=\bar{y}\left(\frac{a \bar{x}^{\prime}+b}{a \bar{x}+b}\right)$ and $t_{r s e}=\bar{y} \exp \left\{\frac{\left(a \bar{x}^{\prime}+b\right)-(a \bar{x}+b}{(a \bar{X}+b)+(a \bar{x}+b}\right\}$

To obtain the bias and MSE of $t_{w d}$, we write

$\overline{\mathrm{y}}=\overline{\mathrm{Y}}\left(1+\mathrm{e}_{0}\right), \overline{\mathrm{x}}=\overline{\mathrm{X}}\left(1+\mathrm{e}_{1}\right), \bar{x}^{\prime}=\overline{\mathrm{X}}\left(1+\mathrm{e}_{1}^{\prime}\right)$

such that

$E\left(e_{0}\right)=E\left(e_{1}\right)=E\left(e_{1}\right)=0$.

$\mathrm{E}\left(\mathrm{e}_{0}^{2}\right)=\mathrm{f}_{1} \mathrm{C}_{\mathrm{y}}^{2}, \quad \mathrm{E}\left(\mathrm{e}_{1}^{2}\right)=\mathrm{f}_{1} \mathrm{C}_{\mathrm{x}}^{2}, \quad \mathrm{E}\left(\mathrm{e}_{1}^{2}\right)=\mathrm{f}_{2} \mathrm{C}_{\mathrm{x}}^{2} \quad \mathrm{E}\left(\mathrm{e}_{0} \mathrm{e}_{1}\right)=\mathrm{f}_{1} \rho \mathrm{C}_{\mathrm{y}} \mathrm{C}_{\mathrm{x}}$

$\mathrm{E}\left(\mathrm{e}_{0} \mathrm{e}_{1}^{\prime}\right)=\mathrm{f}_{2} \rho \mathrm{C}_{\mathrm{y}} \mathrm{C}_{\mathrm{x}} \quad \mathrm{E}\left(\mathrm{e}_{1} \mathrm{e}_{1}{ }_{1}\right)=\mathrm{f}_{2} \mathrm{C}_{\mathrm{x}}^{2}$

where $\quad f_{1}=\left(\frac{1}{n}-\frac{1}{N}\right), \quad f_{2}=\left(\frac{1}{n^{\prime}}-\frac{1}{N}\right)$

Following the procedure mentioned in section 2 and 3, we get bias and MSE of $t_{w d}$ as

$$
B\left(t_{\omega d}\right)=\bar{y}\left[\theta^{2} C_{x}^{2} f_{3}\left(\omega_{1 d}+\frac{3 \omega_{2 d}}{8}\right)-\theta \rho C_{y} C_{x} f_{3}\left(\omega_{1 d}+\frac{\omega_{2 d}}{2}\right)\right]
$$


$\operatorname{MSE}\left(\mathrm{t}_{\omega \mathrm{d}}\right)=\overline{\mathrm{Y}}^{2}\left[\mathrm{f}_{1} \mathrm{C}_{\mathrm{y}}^{2}+\mathrm{f}_{3} \mathrm{C}_{\mathrm{x}}^{2} \omega_{\mathrm{d}}^{2}-2 \mathrm{f}_{3} \rho \mathrm{C}_{\mathrm{y}} \mathrm{C}_{\mathrm{x}} \omega_{\mathrm{d}}\right]$

where $\mathrm{f}_{3}=\frac{1}{\mathrm{n}}-\frac{1}{\mathrm{n}^{\prime}}=\mathrm{f}_{1}-\mathrm{f}_{2}$.

MSE $\left(t_{\omega d}\right)$ is minimum, when

$\omega_{1 \mathrm{~d}}+\frac{\omega_{2} \mathrm{~d}}{2}=\omega_{\mathrm{d}}=\mathrm{k}$

Putting this value of $\omega_{d}$ in (4.4), we get the minimum MSE of $t_{\omega d}$ as

$\min \operatorname{MSE}\left(\mathrm{t}_{\omega \mathrm{d}}\right)=\overline{\mathrm{Y}}^{2} \mathrm{C}_{\mathrm{y}}^{2}\left[\mathrm{f}_{1}-\mathrm{f}_{3} \rho^{2}\right]$

This is same as that of traditional two phase linear regression estimator.

The bias expression for the estimators $t_{\mathrm{rsd}}$ and $t_{\mathrm{rsde}}$ is respectively given by

$B\left(t_{r s d}\right)=\bar{Y}\left[\theta^{2} c_{x}^{2} f_{3} \omega_{1 d}-\theta \rho c_{y} c_{x} f_{3} \omega_{1 d}\right]$

$\mathbf{B}\left(\mathrm{t}_{\mathrm{rsde}}\right)=\overline{\mathrm{Y}}\left[\theta^{2} \mathrm{C}_{\mathrm{x}}^{2} \mathrm{f}_{3} \frac{3 \omega_{2 \mathrm{~d}}}{8}-\theta \rho \mathrm{C}_{\mathrm{y}} \mathrm{C}_{\mathrm{x}} \mathrm{f}_{3} \frac{\omega_{2 \mathrm{~d}}}{2}\right]$

From (4.2) and (4.5), we have only two equations in three unknowns. It is not possible to find the unique values for $w_{\text {id }}$ 's $i=0,1,2$.

In order to get unique values of $w_{i d}$, we shall impose linear restriction

$\omega_{0 d} B(\bar{y})+\omega_{1 d} B\left(t_{r s d}\right)+\omega_{2 d} B\left(t_{r s e d}\right)=0$

Equations (4.2), (4.5) and (4.9) can be written in matrix form, as

$$
\left[\begin{array}{ccc}
1 & 1 & 1 \\
0 & 1 & 1 / 2 \\
0 & B\left(t_{\text {rsd }}\right) & B\left(t_{\text {rsed }}\right)
\end{array}\right]\left[\begin{array}{l}
\omega_{\text {od }} \\
\omega_{1 d} \\
\omega_{2 d}
\end{array}\right]=\left[\begin{array}{l}
1 \\
k \\
0
\end{array}\right]
$$

Solving (4.10), we get the unique values of $\omega_{i d}^{\prime} s$, $(i=0,1,2)$ as

$$
\left.\begin{array}{l}
\omega_{0 \mathrm{~d}}=\frac{\Delta_{\mathrm{od}}}{\Delta_{\mathrm{rd}}} \\
\omega_{1 \mathrm{~d}}=\frac{\Delta_{1 \mathrm{~d}}}{\Delta_{\mathrm{rd}}} \\
\omega_{2 \mathrm{~d}}=\frac{\Delta_{\mathrm{rd}}}{\Delta_{\mathrm{rd}}}
\end{array}\right\}
$$

where

$$
\left.\begin{array}{c}
\Delta_{\mathrm{rd}}=\mathrm{B}\left(\mathrm{t}_{\mathrm{rsed}}\right)-\frac{1}{2} \mathrm{~B}\left(\mathrm{t}_{\mathrm{rsd}}\right) \\
\Delta_{\mathrm{r} 0 \mathrm{~d}}=\mathrm{B}\left(\mathrm{t}_{\mathrm{rsed}}\right)\{1-\mathrm{k}\}+\frac{1}{2} \mathrm{~B}\left(\mathrm{t}_{\mathrm{rsd}}\right)\left\{\mathrm{k}-\frac{1}{2}\right\} \\
\Delta_{\mathrm{r} 1 \mathrm{~d}}=\mathrm{k} \cdot \mathrm{B}\left(\mathrm{t}_{\mathrm{rsed}}\right) \\
\Delta_{\mathrm{r} 2 \mathrm{~d}}=-\mathrm{k} \cdot \mathrm{B}\left(\mathrm{t}_{\mathrm{rsd}}\right)
\end{array}\right\}
$$

Use of these $w_{\text {id }}$ 's $(i=0,1,2)$ will remove the bias up to terms of order $O\left(n^{-1}\right)$ at (4.1). 
The estimator $t_{q}$ written in (3.1), in two phase sampling, will take following form

$\mathbf{t}_{\mathrm{qd}}=\mathrm{q}_{0 \mathrm{~d}} \overline{\mathrm{y}}+\mathrm{q}_{1 \mathrm{~d}} \mathrm{t}_{\mathrm{psd}}+\mathrm{q}_{2 \mathrm{~d}} \mathrm{t}_{\mathrm{psed}} \in \mathrm{Q}_{\mathrm{d}}$

For $\sum_{i=0}^{2} q_{i d}=1, q_{i d} \in R$.

where $q_{i d}(\mathrm{i}=0,1,2)$ denotes the statistical constants .

The estimators $t_{p s d}$ and $t_{p s d e}$ are

$t_{p s d}=\bar{y}\left(\frac{a \bar{x}+b}{a \overline{X^{\prime}}+b}\right)$ and

$$
\mathrm{t}_{\mathrm{psed}}=\overline{\mathrm{y}} \exp \left\{\frac{(\mathrm{a} \overline{\mathrm{x}}+\mathrm{b})-\left(\mathrm{a} \overline{\mathrm{X}}^{\prime}+\mathrm{b}\right.}{(\mathrm{a} \overline{\mathrm{x}}+\mathrm{b})+\left(\mathrm{a} \overline{\mathrm{X}}^{\prime}+\mathrm{b}\right.}\right\}
$$

Following the procedure of section 4 , we get the unique values of qid $_{\text {id }} \mathbf{s}(\mathrm{i}=0,1,2)$ as

$\mathrm{q}_{0 \mathrm{~d}}=\frac{\Delta_{\mathrm{pod}}}{\Delta_{\mathrm{pd}}}$

$\left.\mathrm{q}_{1 \mathrm{~d}}=\frac{\Delta_{\mathrm{p} 1 \mathrm{~d}}}{\Delta_{\mathrm{pd}}}\right\}$

$q_{2 d}=\frac{\Delta_{\mathrm{p} 2 \mathrm{~d}}}{\Delta_{\mathrm{pd}}}$

where

$$
\left.\begin{array}{c}
\Delta_{\mathrm{pd}}=\mathrm{B}\left(\mathrm{t}_{\mathrm{rsed}}\right)-\frac{1}{2} \mathrm{~B}\left(\mathrm{t}_{\mathrm{rsd}}\right) \\
\Delta_{\mathrm{p} 0 \mathrm{~d}}=\mathrm{B}\left(\mathrm{t}_{\mathrm{rsed}}\right)\{1+\mathrm{k}\}+\frac{1}{2} \mathrm{~B}\left(\mathrm{t}_{\mathrm{rsd}}\right)\left\{-\mathrm{k}-\frac{1}{2}\right\} \\
\Delta_{\mathrm{p} 1 \mathrm{~d}}=-\mathrm{k} \cdot \mathrm{B}\left(\mathrm{t}_{\mathrm{psed}}\right) \\
\Delta_{\mathrm{p} 2 \mathrm{~d}}=\mathrm{k} \cdot \mathrm{B}\left(\mathrm{t}_{\mathrm{psd}}\right)
\end{array}\right\}
$$

where

$\mathrm{B}\left(\mathrm{t}_{\mathrm{psd}}\right)=\overline{\mathrm{Y}}\left[\theta \mathrm{q}_{1 \mathrm{~d}} \mathrm{f}_{3} \rho \mathrm{C}_{\mathrm{y}} \mathrm{C}_{\mathrm{x}}\right]$

$\mathbf{B}\left(\mathrm{t}_{\mathrm{psed}}\right)=\overline{\mathrm{Y}}\left[\theta \frac{\mathrm{q}_{2 \mathrm{~d}}}{2} \mathrm{f}_{3} \rho \mathrm{C}_{\mathrm{y}} \mathrm{C}_{x}-\frac{\theta^{2}}{8} \mathrm{q}_{2 \mathrm{~d}} \mathrm{f}_{3} \mathrm{C}_{x}^{2}\right]$

The minimum MSE of $\mathrm{t}_{\mathrm{qd}}$ is given by

$\operatorname{MSE}\left(\mathrm{t}_{\mathrm{qd}}\right)=\overline{\mathrm{Y}}^{2} \mathrm{C}_{\mathrm{y}}^{2}\left[\mathrm{f}_{1}-\mathrm{f}_{3} \rho^{2}\right]$.

\section{Empirical study}

For empirical study we use the data sets earlier used by Kadilar and Cingi (2006) (population 1) and Khosnevisan et. al. (2007) (population 2) to verify the theoretical results. 


\section{Data statistics}

\begin{tabular}{|l|c|c|c|c|c|c|c|c|}
\hline Population & $\mathrm{N}$ & $\mathrm{n}$ & $\overline{\mathrm{Y}}$ & $\overline{\mathrm{X}}$ & $\mathrm{C}_{\mathrm{y}}$ & $\mathrm{C}_{\mathrm{x}}$ & $\rho$ & $\beta_{2}(\mathrm{x})$ \\
\hline Population 1 & 106 & 20 & 2212.59 & 27421.7 & 5.22 & 2.10 & 0.86 & 34.57 \\
\hline Population 2 & 20 & 8 & 19.55 & 18.8 & 0.355 & 0.394 & -0.92 & 3.06 \\
\hline
\end{tabular}

Table 5.1: Values of $\omega_{i}^{\prime}$ s and $q_{i}^{\prime}$ 's

\begin{tabular}{|c|c|c|}
\hline$\omega_{i}$ 's & Population 1 & Population 2 \\
\hline$\omega_{0}$ & 8.590718 & 7.892148 \\
$\left(\mathrm{q}_{0}\right)$ & $(21.417)$ & $(2.919085)$ \\
\hline$\omega_{1}$ & 11.86615 & 5.234461 \\
$\left(\mathrm{q}_{1}\right)$ & $(16.14158)$ & $(3.576773)$ \\
\hline$\omega_{2}$ & -19.4569 & -12.1266 \\
$\left(\mathrm{q}_{2}\right)$ & $(-36.5586)$ & $(-5.49586)$ \\
\hline
\end{tabular}

The percent relative efficiencies (PRE) of various estimators of $\bar{Y}$ are computed and presented in Table 5.2 below.

Table 5.2: PRE of different estimators of $\bar{Y}$

\begin{tabular}{|c|c|c|c|}
\hline Estimator & PRE (Pop I) & Estimator & PRE (Pop II) \\
\hline $\mathrm{t}_{0}$ & 100 & $\mathrm{q}_{0}$ & 100 \\
\hline $\mathrm{t}_{1}$ & 212.816 & $\mathrm{q}_{1}$ & 526 \\
\hline $\mathrm{t}_{2}$ & 212.803 & $\mathrm{q}_{2}$ & 550.261 \\
\hline $\mathrm{t}_{3}$ & 212.606 & $\mathrm{q}_{3}$ & 645.256 \\
\hline $\mathrm{t}_{4}$ & 212.815 & $\mathrm{q}_{4}$ & 534.592 \\
\hline $\mathrm{t}_{5}$ & 212.716 & $\mathrm{q}_{5}$ & 581.732 \\
\hline $\mathrm{t}_{6}$ & 212.810 & $\mathrm{q}_{6}$ & 465.501 \\
\hline $\mathrm{t}_{7}$ & 143.992 & $\mathrm{q}_{7}$ & 384.447 \\
\hline $\mathrm{t}_{8}$ & 143.923 & $\mathrm{q}_{8}$ & 285.920 \\
\hline $\mathrm{t}_{9}$ & 143.988 & $\mathrm{q}_{9}$ & 338.487 \\
\hline $\mathrm{t}_{10}$ & 143.990 & $\mathrm{q}_{10}$ & 374.584 \\
\hline $\mathrm{t}_{11}$ & 143.991 & $\mathrm{q}_{11}$ & 345.118 \\
\hline $\mathrm{t}_{12}$ & 143.959 & $\mathrm{q}_{12}$ & 231.602 \\
\hline $\mathrm{t}_{13}$ & 143.991 & $\mathrm{q}_{13}$ & 424.194 \\
\hline $\mathrm{t}_{14}$ & 143.987 & $\mathrm{q}_{14}$ & 360.086 \\
\hline $\mathrm{t}_{15}$ & 143.992 & $\mathrm{q}_{15}$ & 356.520 \\
\hline $\mathrm{t}_{16}$ & 143.911 & $\mathrm{q}_{16}$ & 467.051 \\
\hline $\mathrm{t}_{\mathrm{w}}(\mathrm{opt})$ & 384.025 & $\mathrm{t}_{\mathrm{q}}(\mathrm{opt})$ & 650.263 \\
\hline
\end{tabular}


In order to see the performance of the suggested estimators in two phase sampling we use the data set of Murthy (1967) (Population III) and Steel and Torrie (1960) (Population IV).

\begin{tabular}{|l|c|c|c|c|c|c|}
\hline Population & $C_{y}$ & $C_{x}$ & $\rho$ & $\mathrm{N}$ & $\mathrm{n}^{\prime}$ & $\mathrm{n}$ \\
\hline Population 1 & 0.3542 & 0.9484 & 0.9150 & 80 & 30 & 10 \\
\hline Population2 & 0.4803 & 0.7493 & -0.4996 & 30 & 12 & 4 \\
\hline
\end{tabular}

Table 5.3: The values of $\omega_{i d}$ 's and $q_{i d}{ }^{\prime} s$

\begin{tabular}{|c|c|c|}
\hline $\begin{array}{c}\boldsymbol{\omega}_{i d} \text { 's } \\
\left(\boldsymbol{q}_{\text {idd }} \boldsymbol{s}\right)\end{array}$ & Population III & Population IV \\
\hline$\omega_{\text {Dd }}$ & -0.241523 & 3.011435 \\
$\left(q_{0 d)}\right)$ & $(1.808833)$ & $(1.089979)$ \\
\hline$\omega_{1 d}$ & -0.558071 & 1.370950 \\
$\left(q_{1 d}\right)$ & $(0.125381)$ & $(0.730464)$ \\
\hline$\omega_{2 d}$ & 1.799595 & -3.382385 \\
$\left(q_{2 d}\right)$ & $(-0.934214)$ & $(-0.820443)$ \\
\hline
\end{tabular}

The percent relative efficiencies of various estimators of $\bar{Y}$ in two phase sampling are computed and presented in Table 5.4 below.

Table 5.4 : PRE of different estimators of $\bar{Y}$ in two phase sampling

\begin{tabular}{|c|c|c|}
\hline Estimator & PRE (Population I) & PRE (Population II) \\
\hline $\bar{y}$ & 100 & 100 \\
\hline $\mathrm{t}_{\mathrm{rsd}}$ & 36.642 & 24.562 \\
\hline $\mathrm{t}_{\mathrm{psd}}$ & 4.849 & 59.770 \\
\hline $\mathrm{t}_{\mathrm{rsde}}$ & 200.420 & 48.365 \\
\hline $\mathrm{t}_{\mathrm{psed}}$ & 23.628 & 115.142 \\
\hline $\mathrm{t}_{\omega \mathrm{d}}$ & 276.156 & 63.452 \\
\hline $\mathrm{t}_{\mathrm{qd}}$ & 34.321 & 123.762 \\
\hline $\mathrm{t}_{\mathrm{opt}}$ & 276.156 & 123.762 \\
\hline
\end{tabular}

\section{Conclusion}

From theoretical discussion and empirical study we conclude that the proposed estimators under optimum conditions perform better than other estimators considered in the article. The relative efficiencies of various estimators are listed in Table 5.2 and 5.4. 


\section{Appendix A}

Table A.1: Some members of the proposed family of estimators -

\begin{tabular}{|c|c|c|c|c|c|c|}
\hline a & b & $\begin{array}{c}\omega_{0} \\
\left(\mathbf{q}_{0}\right)\end{array}$ & $\begin{array}{c}\omega_{1} \\
\left(\mathbf{q}_{1}\right)\end{array}$ & $\begin{array}{c}\omega_{2} \\
\left(\mathbf{q}_{2}\right)\end{array}$ & $\begin{array}{c}\text { Ratio Estimator } \\
\text { (corresponding to } \\
\left.\omega_{i} \mathrm{i}=0,1,2\right)\end{array}$ & $\begin{array}{c}\text { Product Estimator } \\
\text { (corresponding to } q_{i}, \mathrm{i}=0,1,2 \text { ) }\end{array}$ \\
\hline 0 & 0 & 1 & 0 & 0 & $\begin{array}{l}t_{0}=\bar{y} \\
\text { The mean per unit estimator }\end{array}$ & $\begin{array}{l}\mathrm{q}_{0}=\overline{\mathrm{y}} \\
\text { The mean per unit estimator }\end{array}$ \\
\hline 1 & 0 & 0 & 1 & 0 & $\begin{array}{l}t_{1}=\bar{y} \frac{\bar{x}}{\bar{x}} \\
\text { The usual ratio estimator }\end{array}$ & $\begin{array}{l}\mathrm{q}_{1}=\overline{\mathrm{y}} \frac{\overline{\mathrm{x}}}{\overline{\mathrm{x}}} \\
\text { The usual product estimator }\end{array}$ \\
\hline 1 & $C_{x}$ & 0 & 1 & 0 & $\begin{array}{l}\mathrm{t}_{2}=\overline{\mathrm{y}} \frac{\overline{\mathrm{X}}+\mathrm{C}_{\mathrm{x}}}{\overline{\mathrm{x}}+\mathrm{C}_{\mathrm{x}}} \\
\text { Sisodia and Dwivedi (1981) } \\
\text { estimator }\end{array}$ & $\begin{array}{l}\mathrm{q}_{2}=\overline{\mathrm{y}} \frac{\overline{\mathrm{x}}+\mathrm{C}_{\mathrm{x}}}{\overline{\mathrm{X}}+\mathrm{C}_{\mathrm{x}}} \\
\text { Pandey and Dubey (1988) } \\
\text { estimator }\end{array}$ \\
\hline 1 & $\beta_{2}(x)$ & 0 & 1 & 0 & $\begin{array}{l}\mathrm{t}_{3}=\overline{\mathrm{y}} \frac{\overline{\mathrm{X}}+\beta_{2}(\mathrm{x})}{\overline{\mathrm{x}}+\beta_{2}(\mathrm{x})} \\
\text { Singh et. al. (2004) estimator }\end{array}$ & $\begin{array}{l}\mathrm{q}_{3}=\overline{\mathrm{y}} \frac{\overline{\mathrm{x}}+\beta_{2}(\mathrm{x})}{\overline{\mathrm{X}}+\beta_{2}(\mathrm{x})} \\
\text { Singh et. al. (2004) estimator }\end{array}$ \\
\hline$\beta_{2}(\mathrm{x})$ & $C_{x}$ & 0 & 1 & 0 & $\begin{array}{l}\mathrm{t}_{4}=\overline{\mathrm{y}} \frac{\overline{\mathrm{x}} \beta_{2}(\mathrm{x})+\mathrm{C}_{\mathrm{x}}}{\overline{\mathrm{x}} \beta_{2}(\mathrm{x})+\mathrm{C}_{\mathrm{x}}} \\
\text { Upadhyaya and Singh (1999) } \\
\text { estimator }\end{array}$ & $\begin{array}{l}\mathrm{q}_{4}=\overline{\mathrm{y}} \frac{\overline{\mathrm{x}} \beta_{2}(\mathrm{x})+\mathrm{C}_{\mathrm{x}}}{\overline{\mathrm{X}} \beta_{2}(\mathrm{x})+\mathrm{C}_{\mathrm{x}}} \\
\text { Upadhyaya and Singh (1999) } \\
\text { estimator }\end{array}$ \\
\hline$C_{x}$ & $\beta_{2}(\mathrm{x})$ & 0 & 1 & 0 & $\begin{array}{l}\mathrm{t}_{5}=\overline{\mathrm{y}} \frac{\overline{\mathrm{X}} \mathrm{C}_{\mathrm{x}}+\beta_{2}(\mathrm{x})}{\overline{\mathrm{x}} \mathrm{C}_{\mathrm{x}}+\beta_{2}(\mathrm{x})} \\
\text { Upadhyaya and Singh (1999) } \\
\text { estimator }\end{array}$ & $\begin{array}{l}\mathrm{q}_{5}=\overline{\mathrm{y}} \frac{\overline{\mathrm{x}} \mathrm{C}_{\mathrm{x}}+\beta_{2}(\mathrm{x})}{\overline{\mathrm{X}} \mathrm{C}_{\mathrm{x}}+\beta_{2}(\mathrm{x})} \\
\text { Upadhyaya and Singh (1999) } \\
\text { estimator }\end{array}$ \\
\hline 1 & $\rho$ & 0 & 1 & 0 & $\begin{array}{l}t_{6}=\bar{y}\left[\frac{\bar{X}+\rho}{\bar{x}+\rho}\right] \\
\text { Singh and Tailor (2003) } \\
\text { estimator }\end{array}$ & $\begin{array}{l}\mathrm{q}_{6}=\overline{\mathrm{y}}\left[\frac{\overline{\mathrm{x}}+\rho}{\overline{\mathrm{x}}+\rho}\right] \\
\text { Singh and Tailor (2003) } \\
\text { estimator }\end{array}$ \\
\hline 1 & 0 & 0 & 0 & 1 & $\begin{array}{l}\mathrm{t}_{7}=\overline{\mathrm{y}} \exp \left[\frac{\overline{\mathrm{X}}-\overline{\mathrm{x}}}{\overline{\mathrm{X}}+\overline{\mathrm{x}}}\right] \\
\text { Bahl and Tuteja (1991) } \\
\text { estimator }\end{array}$ & $\begin{array}{l}\mathrm{q}_{7}=\overline{\mathrm{y}} \exp \left[\frac{\overline{\mathrm{x}}-\overline{\mathrm{X}}}{\overline{\mathrm{X}}+\overline{\mathrm{x}}}\right] \\
\text { Bahl and Tuteja (1991) } \\
\text { estimator }\end{array}$ \\
\hline
\end{tabular}




\begin{tabular}{|c|c|c|c|c|c|c|}
\hline $\mathbf{a}$ & b & $\begin{array}{c}\omega_{0} \\
\left(q_{0}\right)\end{array}$ & $\begin{array}{l}\omega_{1} \\
\left(\mathbf{q}_{1}\right)\end{array}$ & $\begin{array}{c}\omega_{2} \\
\left(\mathbf{q}_{2}\right)\end{array}$ & $\begin{array}{c}\text { Ratio Estimator } \\
\text { (corresponding to } \\
\left.\omega_{i} \mathrm{j}=0,1,2\right)\end{array}$ & $\begin{array}{c}\text { Product Estimator } \\
\text { (corresponding to } q_{i}, \mathrm{i}=0,1,2 \text { ) }\end{array}$ \\
\hline 1 & $\beta_{2}(x)$ & 0 & 0 & 1 & $\begin{array}{l}t_{8}=\bar{y} \exp \left[\frac{\bar{X}-\bar{x}}{\bar{X}+\bar{x}+2 \beta_{2}(x)}\right] \\
\text { Singh et. al. (2007) estimator }\end{array}$ & $\begin{array}{l}q_{8}=\bar{y} \exp \left[\frac{\bar{x}-\bar{X}}{\bar{X}+\bar{x}+2 \beta_{2}(x)}\right] \\
\text { Singh et. al. (2007) estimator }\end{array}$ \\
\hline 1 & $C_{x}$ & 0 & 0 & 1 & $\begin{array}{l}\mathrm{t}_{9}=\overline{\mathrm{y}} \exp \left[\frac{\overline{\mathrm{X}}-\overline{\mathrm{x}}}{\overline{\mathrm{X}}+\overline{\mathrm{x}}+2 \mathrm{C}_{\mathrm{x}}}\right] \\
\text { Singh et.al. (2007) estimator }\end{array}$ & $\begin{array}{l}q_{9}=\bar{y} \exp \left[\frac{\bar{x}-\bar{X}}{\bar{X}+\bar{x}+2 C_{x}}\right] \\
\text { Singh et.al. (2007) estimator }\end{array}$ \\
\hline 1 & $\rho$ & 0 & 0 & 1 & $\begin{array}{l}\mathrm{t}_{10}=\overline{\mathrm{y}} \exp \left[\frac{\overline{\mathrm{X}}-\overline{\mathrm{x}}}{\overline{\mathrm{X}}+\overline{\mathrm{x}}+2 \rho}\right] \\
\text { Singh et. al.(2007) estimator }\end{array}$ & $\begin{array}{l}\mathrm{q}_{10}=\overline{\mathrm{y}} \exp \left[\frac{\overline{\mathrm{x}}-\overline{\mathrm{X}}}{\overline{\mathrm{X}}+\overline{\mathrm{x}}+2 \rho}\right] \\
\text { Singh et. al.(2007) estimator }\end{array}$ \\
\hline$\beta_{2}(x)$ & $C_{x}$ & 0 & 0 & 1 & $\begin{array}{l}t_{11}=\bar{y} \exp \left[\frac{\left.\beta_{2}(x) \bar{X}-\bar{x}\right)}{\beta_{2}(x)(\bar{X}+\bar{x})+2 C_{x}}\right] \\
\text { Singh et. al. (2007) estimator }\end{array}$ & $\begin{array}{l}q_{11}=\bar{y} \exp \left[\frac{\beta_{2}(x)(\bar{x}-\bar{X})}{\beta_{2}(x)(\bar{X}+\bar{x})+2 C_{x}}\right] \\
\text { Singh et. al. (2007) estimator }\end{array}$ \\
\hline$C_{x}$ & $\beta_{2}(x)$ & 0 & 0 & 1 & $\begin{array}{l}t_{12}=\bar{y} \exp \left[\frac{C_{x}(\bar{X}-\bar{x})}{C_{x}(\bar{X}+\bar{x})+2 \beta_{2}(x)}\right] \\
\text { Singh et. al. (2007) estimator }\end{array}$ & $\begin{array}{l}q_{12}=\bar{y} \exp \left[\frac{C_{x}(\bar{x}-\bar{X})}{C_{x}(\bar{X}+\bar{x})+2 \beta_{2}(x)}\right. \\
\text { Singh et. al. (2007) estimator }\end{array}$ \\
\hline$C_{x}$ & $\rho$ & 0 & 0 & 1 & $\begin{array}{l}t_{13}=\bar{y} \exp \left[\frac{C_{x}(\bar{X}-\bar{x})}{C_{x}(\bar{X}+\bar{x})+2 \rho}\right] \\
\text { Singh et. al. (2007) estimator }\end{array}$ & $\begin{array}{l}q_{13}=\bar{y} \exp \left[\frac{C_{x}(\bar{x}-\bar{X})}{C_{x}(\bar{X}+\bar{x})+2 \rho}\right] \\
\text { Singh et. al. (2007) estimator }\end{array}$ \\
\hline$\rho$ & $C_{x}$ & 0 & 0 & 1 & $\begin{array}{l}t_{14}=\bar{y} \exp \left[\frac{\rho(\bar{X}-\bar{x})}{\rho(\bar{X}+\bar{x})+2 C_{x}}\right] \\
\text { Singh et. al. (2007) estimator }\end{array}$ & $\begin{array}{l}q_{14}=\bar{y} \exp \left[\frac{\rho(\bar{x}-\bar{X})}{\rho(\bar{X}+\bar{x})+2 C_{x}}\right] \\
\text { Singh et. al. (2007) estimator }\end{array}$ \\
\hline$\beta_{2}(x)$ & $\rho$ & 0 & 0 & 1 & $\begin{array}{l}t_{15}=\bar{y} \exp \left[\frac{\beta_{2}(x)(\bar{X}-\bar{x})}{\beta_{2}(x)(\bar{X}+\bar{x})+2 \rho}\right] \\
\text { Singh et. al. (2007) estimator }\end{array}$ & $\begin{array}{l}q_{15}=\bar{y} \exp \left[\frac{\beta_{2}(x)(\bar{x}-\bar{X})}{\beta_{2}(x)(\bar{X}+\bar{x})+2 \rho}\right] \\
\text { Singh et. al. (2007) estimator }\end{array}$ \\
\hline$\rho$ & $\beta_{2}(x)$ & 0 & 0 & 1 & $\begin{array}{l}t_{16}=\bar{y} \exp \left[\frac{\rho(\bar{X}-\bar{x})}{\rho(\bar{X}+\bar{x})+2 \beta_{2}(x)}\right] \\
\text { Singh et. al. (2007) estimator }\end{array}$ & $\begin{array}{l}q_{16}=\bar{y} \exp \left[\frac{\rho(\bar{x}-\bar{X})}{\rho(\bar{X}+\bar{x})+2 \beta_{2}(x)}\right] \\
\text { Singh et. al. (2007) estimator }\end{array}$ \\
\hline
\end{tabular}


In addition to above estimators a large number of estimators can also be generated from the proposed estimators just by putting different values of constants $\omega_{\mathrm{i}}, \mathrm{i}=0,1,2, \mathrm{q}_{\mathrm{i}}, \mathrm{i}=0,1,2$ and $\mathrm{a}$ and $\mathrm{b}$.

\section{Acknowledgements}

The second author (Mukesh Kumar) is thankful to UGC, New Delhi, India, for providing financial assistance. The authors would like to thank the referee for his constructive suggestions on an earlier draft of the paper.

\section{References}

1. Bahl, S. and Tuteja, R.K. (1991): Ratio and product type exponential estimator. Infrm. and Optim. Sci., XII, I, 159-163.

2. Kadilar, C. and Cingi, H. (2006): New ratio estimators using correlation coefficient. InterStat, 1-11.

3. Khoshnevisan,M., Singh, R., Chauhan, P., Sawan, N. and Smarandache, F.(2007): A general family of estimators for estimating population means using known value of some population parameter(s). Far east journal of statistics, 22(2), 181-191.

4. Pandey, B.N. and Dubey, V. (1988): Modified product estimator using coefficient of variation of auxiliary variate. Assam Statistical Rev. 2(2), 64-66.

5. Sen, A.R. (1978): Estimation of the population mean when the coefficient of variation is known. Commun. Stat. Theo. Meth. A (7), 657-672.

6. Singh, H.P. and Kakran, M.S. (1993): A modified ratio estimator using coefficient of variation of auxiliary character. Unpublished.

7. Singh, H.P. and Tailor, R. (2003): Use of known correlation coefficient in estimating the finite population mean. Statistics in Transition, 6, 4, 555-560.

8. Singh, H.P., Tailor, R, Tailor, R. and Kakran, M.S. (2004): An improved estimator of population mean using power transformation. Jor. Ind. Soc. Agri. Statist. 58(2), 223-230.

9. Singh, J., Pandey, B.N. and Hirano, K. (1973): On the utilization of a known coefficient of kurtosis in the estimation procedure of variance. Ann. Inst. Stat. Math., 25, 51-55.

10. Singh, R. and Kumar, M. (2009): A note on transformations on auxiliary variable in survey sampling. (to appear in MASA)

11. Singh, R., Cauhan, P., Sawan, N. and Smarandache, F. (2007): Auxiliary information and a priory values in construction of improved estimators. Renaissance High press, USA. 
12. Singh, R., Singh, J. and Smarandache, F. (2009): Studies in statistical inference, sampling techniques and demography. ProQuest Information \& Learning, USA.

13. Sisodiya, B. V. S. and Dwivedi, V.K. (1981): A modified ratio estimator using coefficient of variation of auxiliary variable. Jour. Ind. Soc. Agri. Stat., 33, 13-18.

14. Sukhatme, P.V., Sukhatme, B.V., Sukhatme, S. and Ashok, C. (1984): Sampling theory of surveys with applications. lowa State University Press, USA.

15. Upadhyaya, L.N. and Singh, H.P. (1984): On the estimation of the population mean with known coefficient of variation. Biom. Jour., 26(8), 915-922. 